\title{
Análisis de las técnicas de enseñanza-aprendizaje para la elaboración de un protocolo terapéutico en infantes escolarizados con Síndrome de Down
}

\author{
Analysis of teaching-learning techniques for the elaboration of a therapeutic protocol in \\ infants enrolled with Down's Syndrome
}

\begin{abstract}
Análise de técnicas de ensino-aprendizagem para a elaboração de um protocolo terapêutico em lactentes com Síndrome de Down
\end{abstract}

Jessica Prato ${ }^{\mathrm{a}}$,Viviana Hernández ${ }^{\mathrm{b}}$,Mónica Fuentesc, Audin Aloiso Gamboa Suarez*d

${ }^{\mathrm{a} E s p e c i a l i s t a ~ e n ~ O r i e n t a c i o ́ n ~ V o c a c i o n a l ~ y ~ O c u p a c i o n a l-~ U n i v e r s i d a d ~ d e ~ S a n t a n d e r ~(C u ́ c u t a, C o l o m b i a ~) ~(I D), ~ b a ́ s t e r ~ e n ~}$ Intervención Social en las Sociedades del Conocimiento- Universidad de Santander (Cúcuta,Colombia), (ID) ${ }^{\mathrm{E} E s p e c i a l i s t a}$ en gerencia de Empresas, - Universidad de Santander (Cúcuta,Colombia ); (ID) Doctor en Ciencias de La Educación, Universidad Francisco de Paula Samtander-Cúcuta, Colombia.(ID)

Forma de citar: Prato, J., Hernández, V., Fuentes, M. \& Gamboa, A. (2018 Análisis de las técnicas de enseñanzaaprendizaje para la elaboración de un protocolo terapéutico en infantes escolarizados con Síndrome de Down.

Perspectivas, 3(1). 85-101

Recibido: junio 10 de 2017

Aceptado: septiembre 05 de 2017

\section{Palabras clave}

Gesto gráfico, proceso de enseñanza, protocolo terapéutico, síndrome de Down.

\footnotetext{
* Autor para correspondencia audingamboa@ufps.edu.co
}

Resumen: La comunicación es una de las habilidades sobre las cuales se fundamenta la construcción de sociedades pues por su intermedio se organizan individualmente el pensamiento y las ideas y se realiza el intercambio colectivo de ideas, la puesta en común de opiniones (diversas o encontradas) todo lo cual permite activar el complejo entramado de las relaciones sociales. Entre todas las prácticas humanas de comunicación el lenguaje constituye sin duda alguna el sistema más perefecto y acabado, pues al tiempo que permite la elaboración de tipos de pensamiento complejo faculta asimismo su socialización. Aunque el desarrollo lingüístico acompaña a toda persona a lo largo de su vida, se trata de facultad que se activa naturalmente de manera temprana en el periodo crítico de la primera infancia. En el caso de las personas con Síndrome de Down suelen presentarse dificultades en el desarrollo lingüístico, tanto en su vertiente oroal como en la escrita, lo cual se convierte en una barera en su proceso de adaptación social y formación académica. Una de estas dificultades tiene que ver con el desarrollo de destrezas en el gesto gráfico. En esta dirección, la intención del presente estudio, de naturaleza documental, ha consistido en establecerunasíntesissobre un conjunto de indicadores relacionados con técnicas de enseñanzaaprendizaje, destrezas de ejecución y el uso de objetos y elementos

https://doi.org/10.22463/25909215.1426 


\section{Keywords}

Graphic gesture, teaching process, therapeutic protocol, Down syndrome.

\section{Palavras chave}

Gesto gráfico, processo de ensino, protocolo terapêutico, síndrome de Down. para el desarrollo del gesto gráfico en un protocolo de intervención terapútica en infantes escolarizados con síndrome de Down. Los resultados representan un conjunto de indicadores y estrategias de referencia para los profesionales que están a cargo de la intervención terapeútica. En este protocolo se detallan las orientaciones y acciones a seguir en relación con cada uno de los aspectos vinculados con el desarrollo del gesto gráfico en niños con Síndrome de Down.

Abstract: Communication is one of the skills on which the construction of societies is based, because through it, thought and ideas are individually organized and the collective exchange of ideas, the sharing of opinions (diverse or found), is carried out. which allows to activate the complex network of social relations. Among all human practices of communication, language undoubtedly constitutes the most perfect and finished system, since at the same time it allows the elaboration of complex types of thought, it also favors its socialization. Although linguistic development accompanies every person throughout their life, it is a faculty that naturally activates itself early in the critical period of early childhood. In the case of people with Down Syndrome there are usually difficulties in linguistic development, both in its gold and written aspects, which becomes a barrier in the process of social adaptation and academic training. One of these difficulties has to do with the development of skills in the graphic gesture. In this direction, the intention of the present study, of documentary nature, has been to establish a synthesis on a set of indicators related to teaching-learning techniques, execution skills and the use of objects and elements for the development of the graphic gesture in a protocol of therapeutic intervention in infants enrolled with Down syndrome. The results represent a set of indicators and reference strategies for the professionals who are in charge of the therapeutic intervention. This protocol details the guidelines and actions to be followed in relation to each of the aspects related to the development of the graphic gesture in children with Down Syndrome.

Resumo: A comunicação é uma das habilidades em que as sociedades de construção são fundadas, porque através deles pensamento organizado individualmente e ideias e troca coletiva de ideias, partilha de opiniões (diferente ou encontrados) é feito tudo o que permite ativar a complexa rede de relações sociais. Entre todas as práticas humanas de comunicação, a linguagem constitui, sem dúvida, o sistema mais perfeito e acabado, pois ao mesmo tempo permite a elaboração de tipos complexos de pensamento, favorece também a sua socialização. Embora o desenvolvimento linguístico acompanhe todas as pessoas ao 
longo da vida, é uma faculdade que se ativa naturalmente no início do período crítico da primeira infância. No caso das pessoas com Síndrome de Down, geralmente há dificuldades no desenvolvimento linguístico, tanto no aspecto ouro quanto no escrito, o que se torna uma barreira no processo de adaptação social e formação acadêmica. Uma dessas dificuldades tem a ver com o desenvolvimento de habilidades no gesto gráfico. Neste sentido, a intenção deste estudo, documentário na natureza, consistia em estableceruna síntesissobre um conjunto de indicadores relacionados a técnicas de ensino e aprendizagem, habilidades de desempenho e uso de objetos e elementos para o desenvolvimento de gesto gráfico em um protocolo de intervenção terapêutica em lactentes com síndrome de Down. Os resultados representam um conjunto de indicadores e estratégias de referência para os profissionais responsáveis pela intervenção terapêutica. Este protocolo detalha as diretrizes e ações a serem seguidas em relação a cada um dos aspectos relacionados ao desenvolvimento do gesto gráfico em crianças com Síndrome de Down.

\section{Introducción}

El niño con Síndrome de Down posee una anomalía que se genera por la presencia de 47 cromosomas en lugar de los 46 que se encuentran normalmente en una persona. Esta distribución atípica de los cromosomas, con la adición de un cromosoma suplementario (tres en lugar de dos en el par 21, llamada también trisomía 21) constituye el Síndrome de Down.

La alteración cromosómica señalada constituye la causa principal de ciertas alteraciones fisioanatómicasen la persona que la porta,tales como cabeza pequeña y aplanada, orejas reducidas, extensión limitada del cuello, piernas y brazos cortos en comparación con la longitud del tronco, manos pequeñas con dedos decrecidos, pies anchos y dedos de los pies cortos y tendencia a obesidad ligera. A nivel neuropsicológico, hay un retraso en la mielinización, lo que significa que el desarrollo demanda de mayor tiempo. En lo que respecta al nivel sensorial hay disfunción o perdida de la audición a causa de las dimesiones de las orejas y el menor tamaño del cráneo, problemas visuales como estrabismo, miopía, astigmatismo, hipermetropía y menor frecuencia de conducta exploratoria. En cuanto al componente motor presentan disminución en la semicontraccion muscular, dificultad en el equilibrio y en la habilidad para ejecutar secuenciasrápidas de movimientos. Finalmente, a nivel cognitivo la atención es dispersa, inestable y fatigable, con dificultades en la memoria para recuperar y almacenar información. Igualmente se presentan disfusionesen el lenguaje expresivo y comprensivo, lo que termina generando un retraso en la adquisición del vocabulario y estructuras sintácticas complejas.

Tales características influyen en el desempeño de la vida cotidiana, pero con un mayor impacto en el proceso educativo,que durante la infancia constituye la principal área ocupacional de una persona. Los niños con síndrome de Down, con la ayuda de parientes, maestros y terapeutas, tienen que hacer frente a diferentes retos y actividades relacionadas con la comunicación verbal y el aprendizaje de la lectoescritura. Una apropiada interacción con su entorno y la posibilidad de leer y escribir, en efecto, supone un paso fundamental para la socialización de estos niños, su desarrollo congnitivo y la posibilidad de obtener una formación ajustada a sus condiciones. Como se sabe, en la actualidad una persona con Síndrome de Down puede incluso recibir un grado universitario 
$\mathrm{y}$ desempeñarse laboralmente en profesiones $\mathrm{y}$ oficios muy variados.

En lo que respecta a las dificultades frecuentes entre personas con Síndrome de Dowm relacionadas con la comunicación verbal escrita, es preciso diferenciar las asociadas a lalectura y las específicas de la escritura, pues se trata de competencias distintas para las que se activan mecanismos neuropsicomotores especializados diferenciados.

En el caso de la escritura, muchas de las dificultades de las personas con Síndrome de Dowm tienen que ver con limitaciones motoras asociadas a la representación símbolos, signos y gráficos vinculadas con sus particulararidades anatómicas pero también con nivel de desarrollo cognitivo (que puede ser muy variable de individuo en individuo). Durante el proceso motor de la escritura, se nota frecuentemente la inversión de un esfuerzo mayor que se emplearía normalmente y dificultades para la aprehensión de la herramienta escritora, como también en la proyección del gesto gráfico significativo. Las experiencias de los niños con Síndrome de Down son complejas debido a que requieren de un proceso distinto y más dilatado en el tiempo para adquirir cualquier destrezas de escritura. En efecto, la grafo-motricidad parte de las unidades gráficas que conforman el código escrito del lenguaje infantil (CLI) llamadas grafismos. Sus primeras manifestaciones aparecen en las producciones espontáneas de niños entre 1o18-24 meses, pero en la escuela, primero con las actividades de apresto y luego con la enseñanza de la lengua escrita, cuando se afina y precisa el trazo de tales grafías.

Los grafismos, en efecto, son esquemas de la primera representación del mundo infantil que ponen de manifiesto las experiencias internalizadas que el sujeto tiene con los objetos,la expresión de que capta e interpreta mediante los analizadores perceptivos rasgos propios de la escritura taes como la linealidad, la angulosidad, la estructura curvilínea o la redondez. Sin embargo, estas realizaciones no constituyen todavía grafemas y por tanto no existe una conservación cognitiva de sus formas porque no son conceptos, sino soloo imágenes mentales o esquemas. Luego, es evidente la necesidad que presentan los niños con síndrome de Down para adquirir las habilidades grafomotoras,queson de vital importancia para el desarrollo de la preescritura.

Dado el perfil específico de dificultades en el área del lenguaje y la persistencia de muchas de ellas en el tiempo, es necesario que los niños con síndrome de Down cuenten con un apoyo especializado desde el inciode su proceso escolar para desarrollar las habilidades de escritura. Este apoyo no debe seguir los cánones del proceso tradicional de enseñanza, sino adaptarse al sujeto objeto aprendizaje. En este sentido, debe ser visto más bien como un proceso terapéutico perfectamente estructurado en el cual la planificación de las diversas actividades deben estar didácticamente diseñadas teniendo en cuenta todas las consideraciones terapéuticas demandas por las características propias de los escolares con esta condición. En el desarrollo de habilidades de escritura de los niños con Síndrome de Down se debe integrar un trabajo multidisciplinar del que participen docentes, padres de familia y terapeutas ocupacionales. Dicha articulación garantizará la potencialización de las habilidades de escritura dentro del marco de un adecuado protocolo terapéutico.

En el caso colombiano, el actual sistema educativo se ajusta al enfoque inclusivo, que se basa en la valoración de la diversidad como elemento enriquecedor del proceso enseñanza-aprendizaje $y$, en consecuencia, favorecedor del desarrollo humano. Pero el concepto de diversidad nos remite al hecho de que todos los estudiantes tienen necesidades educativas comunes (compartidas por la mayoría) $\mathrm{y}$ unas necesidades propias (individuales), y que algunas de estas necesidades pueden ser especiales. Si en una institución educativa no se cuenta con el personal idóneo para orientar los procesos inclusivos difícilmente se puede llegar a garantizar el aprendizaje significativo del escolar.

Wishart (2002) citado por Murillo(s.f.) sugiere que el proceso de desarrollo de los niños con 
síndrome de Down es fundamentalmente diferente, tanto en su naturaleza como en su curso:

La autora supone que si esto es así, entonces al momento de diseñar y llevar a la práctica los programas de atención temprana $\mathrm{y}$ otras intervenciones educativas se debería tener en cuenta las diferencias en el estilo de aprendizaje natural de los niños y en las vías por las que discurre su desarrollo. De acuerdo con lo anterior se deduce que los niños con síndrome de Down, debido a sus particularidades cognitivas y sensorio motoras presentan un nivel de desarrollo distinto del estándar según sus rango de edad y grado académico.

En relación con la atención de la población con necesidades especiales de educación, Crosso (2010) menciona que en América Latina y el Caribe, de acuerdo con datos del Banco Mundial, solo entre el $20 \%$ y el $30 \%$ de los niños/as con discapacidad asiste a la escuela y que stos, cuando entran, suelen ser excluidos enseguida debido a que se ejerce sobre ellos las mismas prácticas de formación y evaluación practicadas con de los alumnos restantes. En Colombia, solo el 0,32\% de los alumnos que asisten a la escuela tienen alguna discapacidad, promedio muy por debajo del porcentaje de niños y niñas con discapacidad en el país, lo que supone una exclusión de las personas con necesidades especiales del sistema educativo oficial. Las cifras son similares en Argentina (0,69\%) y en México $(0,52 \%)$, mientras que en Uruguay y Nicaragua se informan porcentajes un poco superiores $(2,76 \%$ y $3,5 \%$, respectivamente). Todas estas cifras provienen del Monitoreo Internacional de los Derechos de las Personas con Discapacidad. Lo anterior puede tener como una posible causa el hecho de que en las instituciones educativas no se cuentan con profesionales de terapia ocupacional que ofrezcan guías de orientación de inclusión educativa que permitan integrar o incluir niños en condición de discapacidad en las aulas de clase y gocen de su derecho a la educación.

Según el perfil formativo $\mathrm{y}$ de ejercicio profesional, el terapeuta ocupacional es, entre otros aspectos, una personaespecializada y sensibilizada en el desarrollo de actividades investigativas orientadas hacia la búsqueda, renovación y desarrollo del conocimiento científico aplicable dentro del campo de sus actividades, para el estudio de problemáticas y planteamiento de soluciones que beneficien a la profesión, al individuo y a la comunidad en general. En este contexto, con esta investigación se pretende establecer cuáles son las técnicas de enseñanza-aprendizaje y las destrezas de ejecución para la elaboración de un protocolo terapéutico de gesto grafico significativo en infantes con Síndrome de Down escolarizados de 4 a 6 años.

Para darrespuestaala pregunta deinvestigación, se han considerado diversos antecedentes, dentro de los que puede mencionarse el trabajo realizado por Ruiz (2004) sobre la escolarización de niños y jóvenes con síndrome de Down. El autor ha reunido las preguntas que se hacen con mayor frecuencia, de carácter eminentemente práctico, y las responde desde su experiencia personal, afirmando ser partidario de la integración de los escolares con síndrome de Down en centros ordinarios. Al mismo tiempo reconoce, sin embargo, que la clave del éxito pasa por la aceptación incondicional del alumnado promovido desde el apoyo preciso y concreto del docente y gracias ala flexibilidad de los modelos elegidos de integración escolar. El abordaje exploratorio y cualitativo con el que se realizó la investigación permitió conocer diferentes percepciones y actitudes frente a la presencia de estos estudiantes en el aula regular y como estas pueden incidir en la construcción de identidad de los mismos. Este autor concluye su exposición con la refelxión acerca de la inconveniencia de prácticas exclusivas, tanto para los alumnos con discapacidda como para el resto de sus compañeros, a quienes se niega la experiencia de apertura a la diversidad como modo de conforntarparadigmas y modelos tradicionales no siempre apropiados.

Rodríguez (s.f.) establece diferentes posturas a favor y en contra de la inclusión de alumnos con discapacidades en centro ordinarios. No obstante, la apoya y la considera la actuación más apropiada, siempre y cuando se adopten las medidas pertinentes 
para responder a sus necesidades. Además, asegura que dicha inclusión contribuye al desarrollo integral del resto del alumnado pues favorece en ellos la incorporación y acrecentamiento de actitudes tolerantes, de respeto y de aceptación de la diferencia como algo natural entre las personas.

Fernández y Buceta (2003) manifiestan, siguiendo la información proporcionada por la Fundación Iberoamericana Down, las personas portadoras de este Síndrome pueden presentar problemas de inmunidad, lo que ocasiona una mayor susceptibilidad a infecciones. También suelen ser frecuentes, por un lado, problemas visuales como cataratas congénitas (frecuencia del 4\%) o adquiridas (30-60\%) y errores de refracción (50\%) $\mathrm{y}$, por otro, los trastornos de audición (38-75\%) y la otitis serosa del oído medio (50-70\%). Alrededor del $44 \%$ de las personas con trisomía 21 presentan una cardiopatía congénita y el 31\% obstrucciones respiratorias durante el sueño que se hicieron evidentes en el trabajo de Marichal (2017). Aunque se trata de un ainvestigación general sobre las condiciones de salud de las personas con Síndrome de Down, conviene tener en cuenta las disfunciones perceptuales y cognitivas que pueden influir en el aprendizaje de lalengua escrita.

De la Iglesia y Cancela (2003) señalan que en un bajo porcentaje de alumnos con Síndrome de Down pueden aparecer trastornos de conducta, de la personalidad, neuróticos y esquizofrenia. Con mayor prevalencia (y en consecuencia, con mayor necesidad de cuidado y atención) aparece en un lato porcentaje trastornos tales como depresión y demencia presenil, es decir que las personas que presentan una discapacidad cognitiva posiblemente presenten trastornos adjuntos que dificultan aún más llevar una vida normal.

Díaz-Caneja y Ruiz (2010, citados por Ruiz, 2012), consideranqueenlas adaptacionescurriculares necesarias para la escolarización de niños/as con síndrome de Down, un error pensar directamente en la modificación de los objetivos y contenidos del currículo sin plantearse previamente otro tipo de medidas como las referidas al acceso al currículo o a las adaptaciones curriculares no significativas. En este sentido, los autores señalan una serie de pautas que deben ser tenidas en cuenta en el proceso educativo con niños y niñas con síndrome de Down. El Departamento de Educación, Universidades e Investigación del Gobierno Vasco (2009), propone por su parte una serie de recomendaciones para mejorar el ambiente académico del alumno, tales como crear un proceso de enseñanza-aprendizaje de carácter activo, participativo, creativo, que parta de las capacidades del alumno, así como diseñar actividades cooperativas, basadas en los intereses y la experimentación del alumnado, agrupaciones flexibles y combinar las actividades colectivas con las individuales, criterios que coinciden con las orientaciones metodológicas dadas por la normativa del currículo de educación infantil en España.

En lo que a la memoria se refiere, como iniciación en las estrategias de escritura se debe trabajar en primer término la grafomotricidad, la motricidad fina y el lenguaje. En el trabajo de Silos Suárez (2015) se señala la importancia de iniciar al niño de forma temprana en la lectoescritura para favorecer el lenguaje. A pesar de recibir refuerzo individual, no debemos olvidar que se aprende a hablar hablando, a leer leyendo y a escribir escribiendo, por lo quese deben planificar y desarrollar constantemente actividades que desarrollen todas las vertientes del lenguaje. Por otra parte, es necesario evitar realizar continuamente correcciones directa y promover más bien de manera constante los modelos que se quiere que el alumno aprehenda. La comunicación de los niños con Síndorme de Dowmmejora si se utilizan apoyos visuales, cuando se escriben las indicaciones $\mathrm{u}$ órdenes en la pizarra, poniendo indicadores visuales para identificar espacios, diferentes momentos de la jornada diaria o para conocer las actividades.

Ruiz Rodríguez (2004), por su parte, evidenció las dificultades que estos niños presentan en los problemas de cálculo, manejo de conceptos abstractos, lógica y relaciones entre objetos; este autor sugiera a partir de sus resultados que se debe trabajar la orientación espacial, las propiedades 
de los objetos (forma, tamaño, color o textura), la psicomotricidad como medio para abordar la orientación espacial y temporal y trabajar los conceptos mediante la experimentación y manipulación.

En el contexto de América Latina y el Caribe se han venido promoviendo durante las últimas décadas transformaciones significativas en el ámbito de la educación especial, que se reflejan en la puesta en marcha de procesos de reforma educativa, orientadas fundamentalmente a mejorar la calidad de la educación con equidad y la eficiencia de la totalidad del sistema educativo.

Romero Pérez y Pereira Domínguez (2011) afirman que tales esfuerzos de renovación educativa persiguen, por una parte, adecuar las respuestas del sistema a las demandas de un mundo moderno, caracterizado por acelerados y profundos cambios en el conocimiento y el mercado del trabajo y por otra, intentan superar el amplio fracaso escolar experimentado por los sistemas tradicionales de educación, que se expresan en altos índices de bajo rendimiento, repitencia y deserción escolar. En este sentido, el mayor desafío que enfrenta en la actualidad, la mayoría de los países es cómo hacer efectivo el derecho que tienen todos los niños y jóvenes (incluidos quienes sufren alguna discapacidad) de acceder a la educación y beneficiarse de una enseñanza de calidad adecuada a sus necesidades individuales de aprendizaje. Para progresar hacia la consecución de este objetivo, la escuela ha de conseguir el difícil equilibrio de ofrecer una respuesta educativa, a la vez comprensiva y diversificada, proporcionando una estructura curricular común a todos los alumnos, incluidos aquellos que presentan necesidades educativas especiales (NEE), que evite la discriminación y desigualdad de oportunidades y considere al mismo tiempo sus características y necesidades individuales.

Estos planteamientos han sido recogidos y ratificados por los estados del mundo en una serie de reuniones y declaraciones internacionales dentro de las cuales se destacan, por ser las más relevantes, la Conferencia Mundial de Educación para Todos (Jomtien,1990), la Conferencia Mundial sobre Necesidades Educativas Especiales: Acceso y Calidad; (Salamanca, 1994) y la VII Reunión de Ministros de Educación de la Región de América Latina y el Caribe (Kingston, 1996).

Dentro del desarrollo de la investigación se hizo necesario realizar una ciertas definiciones que permitieran orientar el fin perseguido sin caer en la ambieguedadde las expresiones citadas. En este sentido se propusieron los siguientes conceptos:

Destrezas de ejecución: son acciones observables, concretas, dirigidas hacia una meta que utiliza el cliente para participar en las ocupaciones de la vida diaria. Ávila et al (2010) también definieron estas destrezas como unidades pequeñas y medibles en una cadena de acciones que son observadas mientras la persona lleva a cabo tareas significativas. Estas son aprendidas y desarrolladas a través del tiempo y son ubicadas en contextos y entornos específicos.

Gesto gráfico: la escritura espontánea de un mismo autor evidenciará una lógica variación en sus características morfocinéticas, dentro de un cierto rango, mayor o menor según los individuos. Para conocer el gesto gráfico de una persona, es necesario captar un gran número de elementos, tales comovelocidad, ritmo, angulosidad, orden, regularidad, dimensión, dirección e inclinación, todo lo cual integra el concepto de gesto Gráfico como conjunto de fenómenos personales que se producen en la escritura y que varía, lógicamente, de persona a persona (Vallejos, 2010).

Técnicas de enseñanza-aprendizaje: se entiende por técnica al conjunto de procedimientos, tácticas o recursos de los que se vale una ciencia, arte, un oficio o una profesión. Cuando se habla de educación, una técnica de enseñanza es un tipo de acción concreta, planificada por el docente y llevada a cabo por el propio docente y/o sus estudiantes con la finalidad de alcanzar objetivos de aprendizaje.

Demandas de la actividad: se refieren a las características específicas de una actividad que influyen en el tipo y cantidad de esfuerzo requerido para realizar tal actividad. Los profesionales 
de terapia ocupacional analizan las actividades para comprender lo que se requiere del cliente y determinar la relación de los requisitos de la actividad para participar de la ocupación.

En lo que respecta al marco normativo que regula este proceso investigativo se destaca la Ley 949 del 2005 mediante la cual se dictan las normas para el ejercicio de la profesión de Terapia Ocupacional en Colombia. También debe nombrarse la Ley 115 de febrero 8 de 1994, en la que se señalan las normas generales para regular el Servicio Público de la Educación, que debe cumplir una función social acorde con las necesidades e intereses de las personas, de la familia y de la sociedad. Finalmente, en Colombia está en vigor la Ley 1618 de 2013 mediante la cual el Estado pretende garantizar y asegurar el ejercicio efectivo de los derechos de las personas con discapacidad mediante la adopción de medidas y políticas que cuenten con enfoque de inclusión, lo que permitirá eliminar toda forma de discriminación en el país por cuenta de una condición.

Dado que los procesos terapéuticos están intrínsecamente relacionados con los procedimientos y protocolos debidamente estandarizados y que por lo general provienen de distintos contexto, se considera primordial establecer un criterio unificado que sirva de referencia en el ejercio profesional de los terapeutas. En consecuencia, en el presente estudio se pretende sintetizar un conjunto de indicadores relacionados con técnicas de enseñanza-aprendizaje, destrezas de ejecución y el uso de objetos y elementos para el desarrollo del gesto gráfico en un protocolo de intervención terapútica en infantes escolarizados con síndrome de Down.

\section{Método}

\subsection{Naturaleza del estudio}

En la presente investigación, de naturaleza documental, se toma como fuente primaria la literatura académica y científica en torno al uso y puesta en práctica de protocolos terapéuticos relacionados con las técnicas de enseñanzaaprendizaje del gesto gráfico, las destrezas de ejecución y el uso de objetos y elementos para el fortalecimiento de esta habilidad.La elaboración del referido protocolo se fundamentó en una investigación de nivel descriptivo con diseño documental (Arias, 2012). La investigación se desarrolló en varias etapas:En primer lugar se inició la búsqueda de información para orientar la construcción del Protocolo del Gesto Gráfico Significativo en infantes con síndrome de Down escolarizados de 4 a 6 años. Posteriormente, se aplicaron técnicas relacionadas con la codificación temática para identifica los indicadores y estrategias que sirven de referencia a los terapeutas para el manejo de escolares con esta condición.

\subsection{Muestra}

La población objeto de estudio está consitituida por todos aquellos trabajos, investigaciones o documentos de carácter académico que conforman la temática de estudio: Síndrome de Down. De cada documento se extrajeron los contenidos relacionados con las técnicas de enseñanza - aprendizaje, destrezas de ejecución y el uso de elementos para el desarrollo del gesto gráfico y la formulación del protocolo terapéutico.

\subsection{Recolección de la información}

Para la recolección de los datos se aplicó la técnica del fichaje; concretamente se utilizó la ficha bibliográfica y los datos aportados en las diferentes fuentes documentales se plasmaron en la matriz de sistematización en la cual se registró la referencia de las fuentes consultadas, la síntesis de los temas y temáticas indagadas, se establecieron las semejanzas y diferencias entre los documentos y se arribó finalmente a las conclusiones.

\subsection{Procedimientos de análisis}

Luego de ubicados, depurados y roganizados, al conjunto de datosse aplicó la técnica del 
subrayado para identificar las ideas principales y secundarias relacionadas con los objetivos de investigación. Después de una segunda lectura con mayor profundidad, se interpretó cada una de las categorías analizadas y se procedió a construir cuadros y gráficos analíticos para sistematizar los resultados de la investigación.

Las técnicas utilizadas para el análisis de la información fueron de carácter hermenéutico, concretamnte la codificación temática. El modo de proceder fue el siguiente: A partir del texto se hace referencia al contexto del otro y al contexto propio. Por último, a partir de la síntesis integrativa del significado del otro y del contexto propio se realza una síntesis integrativa y se construyen interpretaciones como propuesta de un nuevo significado.
Finalmente, se procedió a construir cada uno de los elementos que conformaron el Protocolo Terapéutico para potencializar el desarrollo del gesto gráfico significativo en niños con síndrome de Down de 4 a 6 años basado en los fundamentos teóricos y conceptuales multidisciplinarios de la pedagogía, la psicología y la terapia ocupacional.

\section{Resultados}

Un primer grupo de resultados está relacionado con la identificación de las diversas técnicas de enseñanza-aprendizaje del gesto gráfico, en las que se referencia el nombre de la técnica y sus respectivas características (ver tabla 1).

Tabla 1

Identificación de las técnicas de enseñanza-aprendizaje gráfico.

\begin{tabular}{|c|c|}
\hline Técnico & Característica de la técnica \\
\hline $\begin{array}{c}\text { Actividades } \\
\text { digitomanuales }\end{array}$ & $\begin{array}{l}\text { Se requiere de dos habilidades: } \\
\text { Actividades manuales. Consiste en abrir y cerrar los puños, como también juntar y separar las manos, rotar sobre las } \\
\text { muñecas presentando las palmas y el dorso. } \\
\text { Actividades digitales. Se opone el pulgar a cada uno de los otros dedos. Es decir, actividades que impliquen el } \\
\text { desarrollo de cada uno de los dedos que permite flexionar y extender. Entre estas actividades se encuentran empunar } \\
\text { la mano y sacar los dedos uno a uno y teclear en la mesa simultaneando los movimientos de los dedos de ambas } \\
\text { manos. }\end{array}$ \\
\hline $\begin{array}{l}\text { Técnicas } \\
\text { pictográficas y } \\
\text { arabescos }\end{array}$ & $\begin{array}{l}\text { Se enfoca en la búsqueda de la distensión motriz y la fluidez del movimiento. } \\
\text { Elaprendizaje técnico se dirige esencialmente a los datos cualitativos y estéticos del trazo y de la superficie de apoyo. } \\
\text { Los arabescos son trazos continuos que no representan un objeto determinadopero facilitan la distensión motriz, el } \\
\text { mejoramiento de las posturas y las reestructuraciones tónicas. }\end{array}$ \\
\hline Rellenado & $\begin{array}{l}\text { Las actividades para desarrollar los trazos se realizan sobre diferentes superficies (suelo, papel de embalar, pizarra, } \\
\text { folios, cuaderno con pauta) y diferentes instrumentos (pinturas de cera, rotuladores, pinceles, lápices, boligrafos). } \\
\text { Los movimientos básicos presentes en los diferentes trazos grafomotores son de dos tipos: rectilineos y curvos, y } \\
\text { sobre ellos se debe centrar la reeducación grafomotriz. } \\
\text { Los ejercicios deben realizarse en sentido izquierda-derecha. } \\
\text { Las actividades para el desarrollo y control de los trazos rectos pueden ser ejercicios de copia en pizarra o papel } \\
\text { cuadriculado: trazado de líneas verticales, horizontales y diagonales, cruces, aspas, paralelas, lineas quebradas, } \\
\text { ángulos y figuras. }\end{array}$ \\
\hline Entramado & $\begin{array}{l}\text { Esta técnica se aplica inicialmente eligiendo una superficie para posteriormente dividirla geométricamente. } \\
\text { El escolar debe rayar regularmente cada una de las zonas resultantes y realizar la misma acción con una orientación } \\
\text { diferente, hasta conseguir un entramado. } \\
\text { Los materiales que se utilizan para esta técnica son el pincel y lápiz de color para más adelante realizarlo con } \\
\text { bolígrafo. }\end{array}$ \\
\hline $\begin{array}{l}\text { Figuras cerradas o } \\
\text { trazos deslizantes }\end{array}$ & $\begin{array}{l}\text { Esta técnica favorece los trazos amplios, a través de los cuales se busca el acercamiento del sujeto a la tarea de la } \\
\text { preescritura. Se intentan favorecer la postura, flexibilidad y la presión sobre la superficie, buscando un desarrollo } \\
\text { regular y rítmico del movimiento. } \\
\text { Los ejercicios deslizantes están relacionados con la relajación. Dentro de ellos se incluyen los grandes trazos } \\
\text { deslizantes, que se clasifican en tres tipos: formas cerradas o semicerradas que el niño imita o repasa sin levantar el } \\
\text { lápiz, las letras de gran formato y los grafismos que van de izquierda a derecha y que abarcan trazados cercanos a la } \\
\text { escritura o a las letras y escritura en gran formato. }\end{array}$ \\
\hline
\end{tabular}




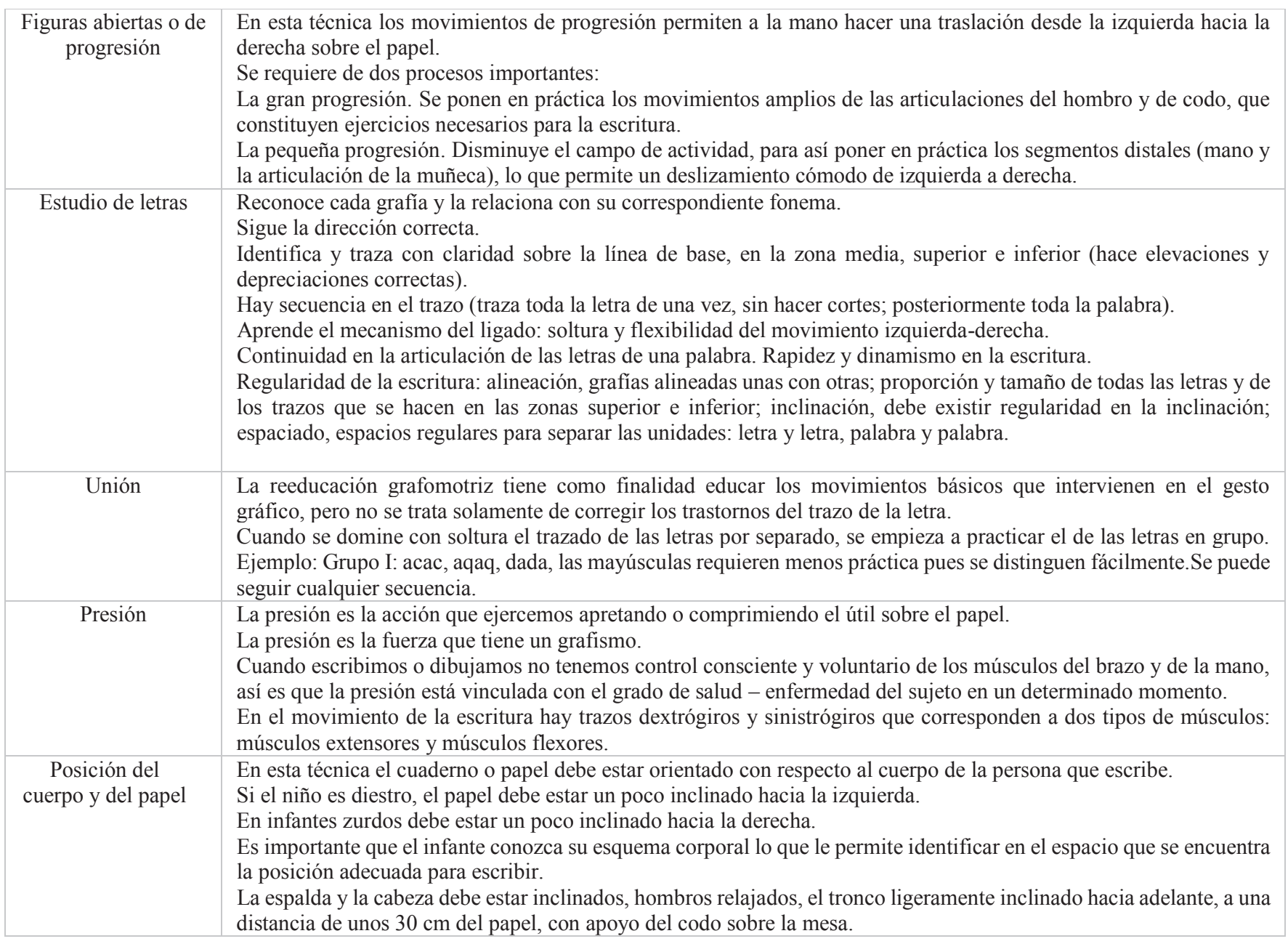

Las técnicas de enseñanza-aprendizaje del gesto gráfico son de vital importancia para la consolidación de las bases del gesto grafico mediante actividades terapéuticas de motricidad fina y grafomotricidad, puesto que por su intermedio se practican la dirección de trazos, la distensión motriz y la postura.

En la tabla 2 se precisan las destrezas de ejecución del gesto gráfico significativo, que incluyen la destreza, el indicador y la síntesis integrativa. Las destrezas de ejecución son de gran importancia dentro del gesto gráfico significativo, dado que a partir de su observación sistemática se puede llevar a cabo la intervención con niños. Para reconocer y atender las necesidades especiales de niños con Síndorme de Down se observan analíticamente las destrezas de ejecución que constituyen el proceso del gesto gráfico, destrezas sensoriales, que incluyen destrezas de naturaleza táctil, el sistema propioceptivo, el sistema visual, las destrezas perceptuales, la cinestesia, el es quema corporal, la discriminación derecha-izquierda, la constancia de forma permite hacer reconocimiento de los objetos y formas en distintos entornos, tamaños y posiciones. La posición en el espacio favorece la percepción que hay entre los objetos hacia sí mismos, lo cual faculta la realización de trazos de arriba abajo, izquierda derecha, diagonal. La figura-fondo permite que infante se ubique en la hoja del cuaderno al momento de hacer el escrito respetando los márgenes del renglón, lo cual permite también reconocer las letras ubicadas en libros. Igualmente es importante el reconocimiento de las destrezas motoras para el gesto gráfico, que 


\section{incluyen el grado de movilidad que permite realizar istrogiros. Asimimso relevantes son las actividades movimientos amplios, los giros, dextrógiros y sin de actividades aprestamiento y procesos escritos.}

Tabla 2

Destrezas de ejecución del gesto gráfico significativo.

\begin{tabular}{|c|c|c|}
\hline \multirow{5}{*}{$\begin{array}{l}\text { Destrezas sensoriales } \\
\text { en niños normotípicos }\end{array}$} & Indicador & Síntesis integrativa \\
\hline & Táctil & $\begin{array}{l}\text { Son acciones o comportamientos que utiliza un cliente para localizar, } \\
\text { identificar y responder a sensaciones y para seleccionar interpretar, asociar, } \\
\text { organizar y recorder eventos sensoriales basado en la discriminación de } \\
\text { experiencias a través de una variedad de sensaciones. Dicho de otro modo, el } \\
\text { sistema somato sensorial es el encargado del registro de las sensaciones, como } \\
\text { los diferentes grados de temperaturas (frío y calor) y la sensibilidad } \\
\text { propioceptiva (posición de los músculos). }\end{array}$ \\
\hline & Propiocepción & $\begin{array}{l}\text { El infante podrá dar respuestas adaptadas a las condiciones del medio o del } \\
\text { entorno. Las habilidades requeridas para el desarrollo del gesto gráfico en las } \\
\text { destrezas sensoriales son las siguientes estás asociadas al sistema sensorial } \\
\text { táctil. Es importante para llegar al gesto grafico puesto que permite la } \\
\text { manipulación de diferentes texturas (líquidas, solidas, blandas, suaves, ásperas, } \\
\text { duras y lisas), las cuales facilitan las actividades de aprestamiento como lo son } \\
\text { el rasgado, rellenado, amasado, recortar, colorear, pegado con diferentes } \\
\text { materiales, de tal modo que se permita al infante desarrollar las destrezas que } \\
\text { se requieren para la utilización de las herramientas escritoras, como el lápiz, } \\
\text { colores y crayones. Dentro del gesto grafico se tiene en cuenta asimismo el } \\
\text { sistema propioceptivo, que está encargado de la interpretación de los estímulos } \\
\text { originados en los músculos, articulaciones y otros tejidos internos que } \\
\text { proporcionan información sobre la posición de una parte del cuerpo en relación } \\
\text { con otras. }\end{array}$ \\
\hline & Visual & $\begin{array}{l}\text { Este sistema sensorial es importante para el desarrollo del gesto gráfico debido } \\
\text { a que proporciona la habilidad para discriminar las partes del cuerpo en relación } \\
\text { con el espacio, la organización postural que se debe tener en el pupitre y la } \\
\text { graduación de la fuerza de contracción que ejercen los escolares para la presión } \\
\text { precisa de la herramienta escritora direccionada hacia la producción gráfica, el } \\
\text { adecuado posicionamiento del lápiz en relación con el escribiente, lo cual } \\
\text { brinda soporte, continuidad y firmeza en la proyección de los trazos; asimismo } \\
\text { el sistema visual faculta la interpretación de estímulos a través de los ojos, que } \\
\text { incluye la visión periférica y la agudeza, reconocimiento de colores y patrones. } \\
\text { Esta destreza es necesaria para la adquisición de las habilidades que requiere el } \\
\text { escolar para ejecutar la proyección gráfica, tales como seguimiento visual en } \\
\text { diferentes direcciones (arriba - abajo, izquierda a derecha), escribir dentro del } \\
\text { renglón respetando limites gráficos, recortar siguiendo una línea, reconocer } \\
\text { figuras y letras. }\end{array}$ \\
\hline & Cinestesia & $\begin{array}{l}\text { La destreza perceptual es la organización de las aferencias sensoriales en } \\
\text { patrones significativos. De tal manera que la interpretación del estímulo } \\
\text { sensorial se percibe a través de los sentidos, abarcando el entorno en el cual se } \\
\text { desenvuelve el escolar. } \\
\text { Dentro de las destrezas perceptuales se halla incluida una variedad de } \\
\text { elementos que forman parte de las habilidades grafomotoras tales como la } \\
\text { cinestesia, que serefiere a la identificación de la cantidad y dirección del }\end{array}$ \\
\hline
\end{tabular}




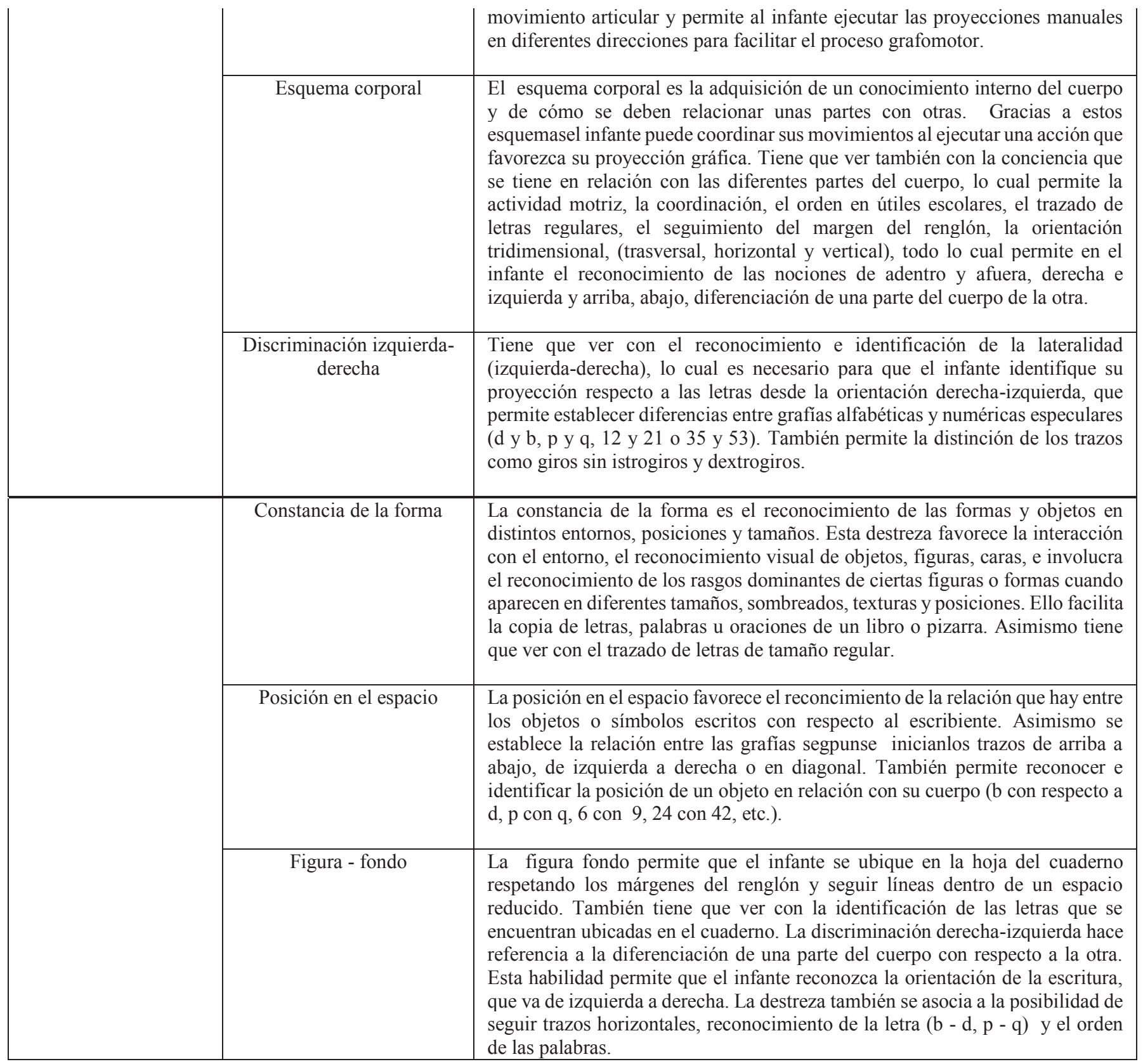

El tono muscular permite la aprehensión de las herramientas escolares, favoreciendo que el infante escriba con mayor fluidez. En la misma dirección, la pulsión tónica facilita proyección del trazo. El control postural y la alineación postural permiten la organización y acomodación del alumno en el pupitre para realizar las actividades. La lateralidad tiene que ver con la dominancia manual en una actividad y diferenciación derecha-izquierda. La integración bilateral implica la habilidad para emplear ambas manos simultáneamente para hacer una actividad, utilizando la mano dominante y la otra como apoyo, El control motriziene que ver con el control de los movimientos y dar dirección a la ejecución de los trazos. La integración visomotriz resulta fundamental en el gestográfico, dado que se debe coordinar el movimiento corporal, en este caso la información visual con la mano, para 
relacionarlas figuras y letras dentro del renglón. Se puede determinar que las destrezas mencionadas son necesarias para el desarrollo del gesto gráfico en los niños en condición de normalidad debido a que son un pilar fundamental en todo lo relacionado con la escritura.

Las destrezas de ejecución y las demandas de la actividad para ejecutar apropiadamente el gesto gráfico significativo son de gran importancia, puesto que brindan información imprescindible para el apropiado abordaje de los porcesos de intervención y apoyo. Para el reconocimeinto de las destrezas de ejecución se inicia con las destrezas sensoriales (indicadores táctiles, discriminación de texturas e identificación del diámetro del objeto) que deberán poner en ejercicio al realizar las actividades manuales. Posteriromenete es preciso atender las destrezas visuales a fin de fortalecer el reconocimiento grafico y la identifiacion de diferentes estímulos cuando se plaman grafías significativas., Entre estas destrezas se encuentra el componetepropioceptivo, que brinda informacion al cerebro sobre la posición y el movimiento de las diferentes partes del cuerpo, facilitándo al infante el posicionamiento y el equlibrio al realizar actividades gráficas manuales.

Asimismo se requiere de destrezas perceptuales. Entre ellas se encuentra la cinestesia, que aporta la consciencia del direccionamiento del movimiento articulardurante el proceso grafomotor. El esquema corporal facilita un conocimieto interno del cuerpo y a su vez las relaciones de las diferentes partes del mismo.

La discriminación derecha izquierda realiza la diferenciación de una parte del cuerpo con respecto a la otra, lo cual es fundamental dentro del gesto gráfico a la hora de efectuar agarres bimanuales de los materiales facilitados. La constancia de la forma permite reconocer las diferentes formas y objetos en distintos entornos y espacios determinados, para que de esta manera el infante realice las manipulaciones dentro de las proyecciones gráficas significativas. La posición en el espacio y la figurade fondo permiten al infante relacionarse con los diferentes objetos y a su vez su reconocimiento.
Cabe mencionar que otra de las destrezas fundamentales dentro del proceso grafomotor tiene que ver, como nompuede ser de otra forma, con las destrezas motoras. El grado de movilidad es fundamental para la proyección gráfica de los infantes, sobre todo si se tiene en cuenta su condición de hiperlaxitudgeneral. El tono muscular, asociada a la contracción parcial, pasiva y continua de los músculos, facilita la proyección manual. La fuerza, por su parte, se refiereal grado de potencia muscular cuando se resiste al movimiento con objetos mediante la gravedad. El control postural y la alineacion postural constituyen la base del desarrollo de habilidades motoras desde un determinante equilibrio, que facilita los movimientos manuales ejercidos.

El cruce de la línea media consiste en el movimiento de los miembros y los ojos a través de un plano sagital, brindándole al infante una adecuada orientación cuando plasmagraficos con significado. La lateralidad permite iniciar los trazos de izquierda a derecha y viceversa.lo cual proporciona al infante seguimiento y direccionamiento de sus miembros al realizar proyecciones gráficas. La integración bilateral coordina los dos lados del cuerpo de modo que uno es apoyo y el otro realiza la acción. El control motriz es necesario para dominar el movimiento y dar dirección adecuada durante la ejecución de los trazos.

Finalmente, las praxias se refieren a los movimientos organizados para llevar a cabo actividades de precisión y velocidad; la coordinacin motora fina es requerida para la manipulación de las diferentes herramientas escolares en las cuales se utilizan pequeños grupos musculares al ejercer la acción. La integración visomotrizconsiste en la coordinación del movimiento corporal: esta coordinación entre la mano y la información visual permite organizar, relacionar las figuras, letras y números y palabras en el espacio dentro del renglón.

Finalmente, en el la tabla 3 se presentan diversos objetos y se describe la estrategia utilizada en su implementación para el trabajo con escolares con Síndrome de Down. 
Tabla 3

Identificación de objetos y su uso.

\begin{tabular}{|c|c|}
\hline Elemento & Estrategia \\
\hline Lápiz & $\begin{array}{l}\text { Las herramientas escritoras como el lápiz, colores, creyones que utilizan los niños con Síndrome de Down } \\
\text { deben tener unas características propias para facilitar el agarre del mismo. Tales características se mencionan } \\
\text { a continuación: la herramienta debe ser gruesa y no tan larga; el peso debe ser liviano; la forma de la } \\
\text { herramienta debe ser hexagonal para facilitar la prensión; la mina del lápiz, color, o crayón debe marcar bien } \\
\text { la hoja de papel para evitar el exceso de presión y que su deslizamiento resulte fácil. Los lápices deben ser al } \\
\text { principio blandos y de mina oscura (5B, 6B, 7B). Posteriormente la herramienta escritora debe tener una punta } \\
\text { más fina para que el niño pueda ver bien el trazo y consiga la precisión necesaria cuando escribe. Durante la } \\
\text { ejecución del gesto grafico en los niños con síndrome de Down de } 4,5 \text { y } 6 \text { años el lápiz debe estar ubicado a } \\
70^{\circ} \text {. }\end{array}$ \\
\hline Hoja & $\begin{array}{l}\text { Las características de la hoja de papel que deben utilizar los niños con Síndrome de Down son las siguientes: } \\
\text { inicialmente la hoja debe ser blanca; no se deben delimitar los espacios; la hoja de papel debe tener unas } \\
\text { medidas aproximadas de } 30 \times 20 \mathrm{~cm} \text { para facilitar los trazos amplios; la hoja se debe ubicar en posición } \\
\text { horizontal para favorecer el seguimiento visual de izquierda a derecha y los movimientos del miembro superior } \\
\text { (mano). }\end{array}$ \\
\hline Silla/mesa & $\begin{array}{l}\text { La silla y la mesa que utilizan los niños con Síndrome de Down debe cumplir con los diferentes requerimientos } \\
\text { que se describen a continuación: debe ser cómoda para que el niño pueda adoptar y mantener una postura } \\
\text { correcta y la estabilidad de troco durante la actividad; la silla no debe estar hundida, ni ser muy blanda; el } \\
\text { fondo de la silla debe tener la misma longitud del muslo; la altura de la silla debe permitir apoyo plantar con } \\
\text { las piernas flexionadas en ángulo recto a la altura de las rodillas. Si el niño no llega completamente al suelo, } \\
\text { se tendrá que poner un soporte debajo de los pies; los brazos deben apoyarse sobre la mesa, con el codo } \\
\text { flexionado de modo que el brazo y antebrazo no queden ni muy altos ni muy bajos. }\end{array}$ \\
\hline Hombro & $\begin{array}{l}\text { El hombro regula principalmente los movimientos de abducción (alejamiento lateral del brazo del eje del } \\
\text { cuerpo) y de aducción (aproximación del brazo al eje del cuerpo). Una de la actividades que se realizan para } \\
\text { estabilizar el hombro en niños con Síndrome de Dow consiste en amasar con diferentes materiales (plastilina, } \\
\text { harina, arcilla, etc.)sobre una mesa amplia. También fortalece estas destrezas pintar sobre la pared en } \\
\text { movimientos amplios, como el trazado de círculos, líneas de arriba abajo, abajo hacia arriba; hacer lanzamiento } \\
\text { de pelota hacia una distancia de } 1 \text { metro, lanzamientos desde arriba y abajo; realizar un tablero, dividirlo en } \\
\text { dos, colocar chinchetas con distancia de } 50 \mathrm{~cm} \text { y luego unirlas con una liga. }\end{array}$ \\
\hline Codo & $\begin{array}{l}\text { Los movimientos realizados en los miembros superiores son necesarios para las actividades de escritura. En el } \\
\text { movimiento del codo se debe tener consciencia de sus límites }\left(90^{\circ} \text { de flexión y } 120^{\circ} \text { de extensión). Sin embargo }\right. \\
\text { los niños con síndrome de Down, presentan dificultades en sus movimientos puesto que son poco coordinados } \\
\text { y a su vez limitados, por ende requieren de apoyo constante para que adquieran mayor habilidad en sus } \\
\text { movimientos, ya sean cortos o amplios al momento realizar grafismos. }\end{array}$ \\
\hline Brazo & $\begin{array}{l}\text { Cuando el niño con síndrome de Down inicia su proceso grafomotor debe tener el brazo y la muñeca apoyada } \\
\text { sobre la mesa, con } 90^{\circ} \text { de flexión, } 120^{\circ} \text { de extensión y } 10^{\circ} \text { de supinación al tomar un objeto. Es importante } \\
\text { observar el modo de coger el lápiz, así como la presión que ejerce en el papel a la hora de trazar las distintas } \\
\text { letras. Para todos los procesos descritos son necesarios los movimientos de los brazos, iniciando con el } \\
\text { dominante sin tomar el no dominante como apoyo. }\end{array}$ \\
\hline Muñeca & $\begin{array}{l}\text { En los niños con Síndrome de Down es importante realizar actividades que permitan la independencia y } \\
\text { segmentación de los movimientos de los dedos con referencia a la mano, para lo cual la mano se debe encontrar } \\
\text { en un rango de movimiento aproximado de } 30^{\circ} \text { de flexión y extensión en la articulación entre el hamate y el } \\
\text { quinto metacarpiano y aproximadamente la mitad, en la articulación entre el hamate y el cuarto metacarpiano. } \\
\text { Este movimiento, junto con la capacidad de flexión de las articulaciones metacarpofalángicas e interfalangicas } \\
\text { del lado cubital, permiten la adaptación para trabajar y posicionar la mano ante la ejecución. Algunas } \\
\text { actividades que se puede realizar son enroscar, rasgar, hacer esferas de papel; tocar instrumentos musicales } \\
\text { como tambor y piano; amasar; actividades de dactilopintura; actividades de lanzamientos con los dedos; dibujar } \\
\text { sobre la arena y ensartar cuentas. }\end{array}$ \\
\hline
\end{tabular}


Otro de los aspectos importantes y a destacar dentro del gesto gráfico significativo tiene que ver con los objetos (y sus propiedades) utilizados para el grafismo. El lápiz, por ejejmplo, debe ser blando, que deje huella fácilmente (para que el niño no se esfuerce innecesariamente en apretar sobre el papel porque no ve lo que traza); los materiales que utilizan los infantes con síndrome de Down deben ser llamativos, manejables y con textura suave. Por ejemplo, la hoja de papel debe cumplir unas características propias para el desarrollo del gesto gráfico. Inicialmente se deben utilizar hojas blancassincuadrículas, en tal caso, sololíneas o algún dibujo,lo cual facilita al escolar la percepción de la figura sobre el fondo.

Dentro de los elementos materiales del aula es necesario para el desarrollo del gesto grafico que la mesa de trabajo se encuentre en disposición cómoda y la silla con el reslporecto, evitando inclinaciones forzadas. La altura de la silla debe permitir que el infante tenga ambos pies apoyados completamente en el suelo (no cruzados o sentados sobre las piernas). Asimismo los brazos deben poder apoyarse sobre la mesa, con el codo flexionado, de modo que el brazo y antebrazo no queden ni muy altos ni muy bajos. Todavía con respecto a las demandas del espacio, el entorno físico que requieren los infantes con síndrome de Down ha de ser amplio, con pocos estímulos distractores tales como los carteleras, dibujos llamativos o colores estridentes. La iluminación debe estar acorde con el espacio en el que se trabaja,ya sea artificial o natural. La ventilación debe proporcionar una temperatura confortable que favorezca la cmodidad en el desarrollo de las actividades académicas. En lo que respecta a la secuencia y el ritmo, los métodos que se utilizan con los infantes con síndrome de Down deben ser claros, cortos, fáciles y llamativos; posterirmente se requiere la interpretación de las funciones y estructuras corporales en los miembros superiores, tales como hombro, codo, brazo y muñeca. Estas estructuras corporales son vitalmente necesarias en el proceso grafomotor del infante, puesto que si llegase a tener limitaciones en algún segmento corporal, su proyección gráfica se verá alterada. También es preciso atender altonomusculardelinfante con Síndorme de Down, pues tiende a ser bajo y limitar, en consecuencia, lamejorfuncionalidad el la proyección gráficasignificativa.

\section{Conclusiones}

Del desarrollo del proceso investigativo a nivel documental se rescatan las siguientes conclusiones:

El gesto gráfico se entiende como el conjunto de los movimientos gráficos realizados con la mano en el acto de la escritura. A través de la educación grafomotora se potencia las habilidades del movimiento gráfico, esencial para la escritura. El mejoramiento de este movimiento es indispensable para que los niños logren una mejor escritura. En consecuencia es fundamental desarrollar en los niños la psicomotricidad fina a través de las técnicas de enseñanza-aprendizaje. De ahí que antes de la aplicación de toda técnica se han de realizar previamente actividades didácticas orientadas al desarrollo de las destrezas de movimiento de las manos y de los dedos y la coordinación visomanual. Esto implica actividades para el desarrollo de las destrezas de manos y dedos, de trazos rectos, de control y de trazos curvos, acompañado de materiales para el trabajo grafomotriz progresivo en un ambiente totalmente lúdico.

En el caso de los niños con Síndrome de Down, al inicio de su proceso de intervención se debe tener en cuenta quédestrezas de ejecucion y demandas de la actividad se requieren para el desarrollo del gesto grafico y como estas influyen en el desarrollo del mismo. En función de estas necesidades se pueden diferenciar funcionalemente las destrezas de ejecución que se requieren para este proceso, siendo fundamentales las sensoriales, perceptuales y motoras para la ejecucion del gesto grafico. Asimismo, en relación con las demandas de la actividad se determinacómo debe ser la herramienta escritora (el lápiz) para una proyección grafica significativay los grados en los que debe estar ubicado el lápiz, al igual que las caracterisicas 
propias que debe tener la hoja que se utiliza para escribir (sin límites gráficos, medidas adecuadas). También resulta relevante la consideración y ajuste entre el mobiliario para la ejecución de actividades escolares, fundaementalmentesillas y mesas y las características antropométricasdel miembro superior (hombro, codo, antebrazo, muñeca y dedos)que convienen para realizar apropiadamente la proyección del trazo.

\section{Referencias}

Arias, F. (2012). El Proyecto de investigación. Introducción a la metodología científica. Caracas: Episteme.

Ávila Álvarez, A.; Martínez Piedrola, R.; Matilla Mora, R.; Máximo Bocanegra, M.; Méndez Méndez, B.; Talavera Valverde, M. A. et al. (2012). Marco de trabajo para la práctica de la terapia ocupacional: Dominio y proceso. $2^{\circ} \mathrm{ed}$ [traducción]. Disponible en: http://www.terapiaocupacional.com/aota2010esp.pdf traducido de: American OccupationalTherapyAsociation (2008). OccupationalTherapyPractice Framework: Domain and Process.

Crosso, C. (2010). El derecho a la educación de personas con discapacidad: impulsando el concepto de educación inclusiva.Revista latinoamericana de educación inclusiva, 4(2), 7995. Disponible en: http://www.repositoriocdpd. net:8080/bitstream/handle/123456789/413/Art CrossoC_DerechoEducacionPersonas_2010. pdf? sequence $=1$

De la Iglesia, J. C. F. y Cancela, M. J. B. (2003). La educación de personas con Síndrome de Down: estrategias de aprendizaje. Santiago de Compostela: Universidade de Santiago de Compostela.

Silos Suárez, A. (2015). Viviendo el deseo de los padres.Reflexión sobre la experiencia de los padres de hijos con discapacidad intelectual. Revista Virtual Canal Down21, 164. Disponible en: https:/www.down21.org/revista-virtual/ revista-virtual-2015/1391-revista-virtual-enero- 2015-numero-164/articulo-profesional-eldeseo-de-los-padres.html

Gobierno Vasco. Departamento de Educación, Universidades e Investigación. (2009). Las competencias básicas en el Sistema Educativo de la Comunidad Autónoma de País Vasco. [Documento en Línea]. Disponible en http://www.euskadi.eus/contenidos/ informacion/dig_publicaciones_innovacion/ es_curricul/adjuntos $/ 14$ _curriculum competencias_300/300002c_Pub_BN_ Competencias_Basicas_c.pdf

Marichal, C. (2017). Inclusión educativa de niños con Síndrome de Down a través de la psicomotricidad. Trabajo de fin de grado de maestro en educación infantil.Tenerife: Universidad de la Laguna. Disponible en: https:// riull.ull.es/xmlui/bitstream/handle/915/5147/ INCLUSION\%20EDUCATIVA \%20DE $\% 20$ NINOS $\% 20 C O N \% 20$ SINDROME $\% 20$ DE $\% 20$ DOW N\%20A\%20TRAVES\%20DE\%20 LA \%20PSICOMOTRICIDAD.pdf?sequence=1

Murillo, M. (2014)Atencion temprana. Niños con síndrome de Down y otros problemas de desarrollo. Madrid: Federación Española del Síndrome de Down. Disponible en http://www.sindromedown.net/wp-ontent/ uploads/2014/09/30L_atenciontemprana.PDF

Rodríguez, A. (2013), La integración e inclusión escolar de los niños con Síndrome de Down. Trabajo final de grado en Educación Primaria. Valladolid: Universidad de Valladolid. Disponible en http:/uvadoc.uva.es/ bitstream/10324/3937/6/TFG-G310.pdf

Romero Pérez, C. y Pereira Domínguez, C. (2011). El enfoque positivo de la educación: aportaciones al desarrollo humano. Teoría de la Educación, 23(2), 69-89.

Ruiz Rodríguez, E. (2012). Programación educativa para escolares con síndrome de Down. Madrid: Publicaciones Down 21. Disponible en: http://www.down21 materialdidactico.org/ libroEmilioRuiz/libroemilioruiz.pdf

Ruiz Rodríguez. E. (2004). La integración escolar 
de los niños con síndrome de Down en España: algunas preguntas y respuestas. Revista síndrome de Down, 21(4), 122-133. Disponible en: http:// revistadown.downcantabria.com/wpcontent/ uploads/2004/12/revista83_122-133.pdf

Rodríguez, E. (2012). Actitudes, estereotipos y prejuicios: su influencia en el síndrome de Down. Revista Síndrome de Down, 29, 110-121. Vallejos. N. (2010). Patrones de confrontación. El cuerpo de escritura. Blog en línea. Disponible

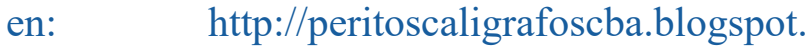
com/2011/07/patrones-de-confrontacion-elcuerpo-de.html 